\title{
Index
}

Activate 17

Activation treatment 25

Active screen plasma 18

Active species 19

Agglomeration 125, 139

Anode 164

Artificial intelligence 68

\section{Battery 153}

Biochar 158

Buckypapers (BP) 81

\section{Carbon 156}

Carbon-based materials 30

Carbon-based polymer nanocomposites 36

Carbon fibers 26-27, 52, 159

Carbon nanofibers 22

Carbon nanomaterial 157

Carbon nanotube (CNT) 34, 81, 115

Carbon nanowalls 159

Carbon paper 23

Catalysts 29

CFRP mirror 81

Chemical vapor deposition 159

Circular economy 66

Class II 129

CNTs 28

Coarse-grained 56

Composite material 27

Coulombic efficiency 173

Deformation experiments 121, 132, 136

Dispersion in nanocomposites 124

Dissipative particle dynamics 55

Effective medium approximation 61

Elastic moduli 48

Electrical conductivity 52, 54, 157

Electrical resistance 20

Electrocatalyst 24

Electrochemical surface area 25

Electrode 155

Electromagnetic compatibility (EMC) 81

Energy storage 154

Energy storage devices 22
Engineered material 66

Epoxy resins 40

Fibre Volume Fraction (FVF) 95

Finite element method 61

Flexible substrates 13

Fuel cells 23

Functional groups 27

Functionalization 30

Functionalize 17

Gas diffusion layer 23

Generalized Hooke's law 62

Glass transition 124

Glass transition temperature 120

Graphene 1, 20, 34, 157

Graphene-based devices 1

Graphene-based nanocomposites 116

Graphene-based polymer nanocomposites 123

Graphene oxide 1

Heterogeneous materials 158

Hydrogen bonds 120, 123, 132, 135-137, 145

In situ polymerization 119

Interfacial characteristics 67

Ion 155

Ion bombardment 19

Kapitza resistance 44

Lattice Boltzmann method 55

Lithium 154

Machine learning 68

Materials Modelling 38

MD algorithm 130

MD simulation packages 131

Melt processing 119

Mesoscale 57

Microscopic structure 67

Model reduction 67

Molecular dynamics 39

Molecular simulations 53, 126-127

Monte Carlo 39

Multiphysics modeling 39 
Nanoscale microfibrillated cellulose 159 Nonequilibrium 40

Nonvolatile memories 1

Nonvolatile resistive memories 6

Optical transparency 20

Passive Intermodulation (PIM) 81

Percolation 54

Phonon 45

Photoresponsive materials 35

Piezoelectric materials 34

Plasma-enhanced CVD (PE-CVD) 29

Plasma-surface interaction 19

Plasma technology 18,30

Platinum nanowires 25

Poly(acrylic acid) 123

Poly(methyl methacrylate) 120

Poly(vinyl alcohol) 120

Polyethylene 122

Polymer electrolyte 165

Polymer matrix composite 26

Polystyrene 121, 132

Polyvinylidene fluoride 122

Potential energy function $126-128,130$

Pouch cell 170

Pyrolysis 159
Raman 161

Recyclable carbon 157

Scotch tape method 116-117

Shape memory materials 33

Smart materials 33

Smoothed particle hydrodynamics 55

Solvent processing 119

Space 82

Space application 13

Specific energy 171

Spray-coating 164

Stress-strain response 48

Surface conductivity 22

Surface engineering 17-18

Surface functionalizations 47

Technology readiness level (TRL) 112

Thermal conductivity 40

Toxicity 68

Tunneling 53

VACNT 29

Waste 157

Wettability 24,28 